
Escape and Avoidance", Proc. of the $45^{\text {th }}$ Annual Meeting of the Human Factors and Ergonomics Society, Minneapolis/St. Paul, MN, October 8-12, 2001.

\title{
ACHIEVING PANACEA: A USABILITY EVALUATION OF A SYSTEM FOR PILOT ALERTING AND NOTIFICATION OF ADVERSE CONDITIONS - ESCAPE AND AVOIDANCE
}

\author{
Patricia May Ververs, Michael C. Dorneich, Michael D. Good \\ Honeywell Laboratories \\ Minneapolis, MN
}

\begin{abstract}
This paper acknowledges the recent thrust to integrate systems on the flight deck and details a usability evaluation of a prototype concept that provides integrated alerting functionality. The ANCOA (Alerting and Notification of Conditions Outside the Aircraft) concept was conceived as means for reducing error conflicts and establishing a clear prioritization among currently independent and disparate alerting systems for hazards external to the aircraft (e.g., TCAS, EGPWS). ANCOA reduces alert proliferation by creating a standardization by which all incoming information is categorized and prioritized under a common framework. By creating a consistent alerting and display philosophy to present information to the crew, we can reduce the demands on pilot attention and information processing. In this paper we discuss a couple of recent integrated hazard awareness systems developed by Honeywell International that make the innovative initial step to incorporate previously separate systems into a single system. We present ANCOA as a concept that can enhance systems of their type and advance their utility. We evaluated the concept's efficacy and present the results of a usability study involving nine commercial pilots that reviewed the concept.
\end{abstract}

Beginning in the mid-1970s when the Ground Proximity Warning System (GPWS) was first introduced, flight safety has vastly improved. Hazard warning systems such as GPWS and Traffic Collision Avoidance Systems (TCAS) provide advanced warnings to the crew concerning hazardous conditions to be avoided. The crew is informed of the impending flight hazard (e.g., terrain, traffic) through visual and aural displays. Additional systems that warn of hazards, such as predictive wind shear, wake vortices, and clear air turbulence, continue to be added or soon will be added to the flight deck. One cause for concern is the sheer number of systems continues to grow. These alerting systems are separate, independent units manufactured by a variety of avionics suppliers, each using different alerting and display philosophies to present information to the crew. Just as the Engine Indicating and Crew Alerting System (EICAS) integrated warnings for systems for internal to the aircraft in the early 1980s (Boucek, et al., 1981), there is a current thrust to do the same for those systems that detect and warn of conditions external to the aircraft.

Honeywell has developed two systems to address the problem of federated information. Two Integrated Hazard Avoidance Systems (IHAS) are available-one for general aviation and the other for the air transport aircraft market. IHAS for general aviation allows either a map, weather, traffic, or terrain information to be displayed as separate pages on a multifunction display. Each function continuously operates in the background regardless of what the crew has selected on the display. Though the general aviation IHAS system should provide improvement over the separate systems, there are several limitations. Since only single parameters (e.g., terrain, traffic, etc.) may be displayed at any one time, the information is not truly integrated. Also the information cannot be overlaid to show relationships among hazards. Additionally, there is no underlying prioritization for the alerts. Therefore, multiple alerts may be triggered without conveying which alert should take precedence.
The IHAS for air transport aircraft is currently under development. It improves on the system available for general aviation by developing an elaborate alert prioritization scheme that resolves concurrent alert signals by determining the condition that is most likely to result in an accident. For instance, reactive wind shear normally has a higher priority than GPWS, which is higher than TCAS. Aural alerts from lower priority events and conditions are inhibited until the alert message of the higher priority hazard is completed.

The ANCOA (Alerting and Notification of Conditions Outside the Aircraft) concept contains components which can build upon IHAS to enhance its functionality. This concept allows multiple sources of information to be overlaid in order to graphically integrate the displayed hazards. Hazards are classified into one of three categories: time-critical, tactical and strategic. ANCOA broadens the scope of current systems by including strategic information. The goal is to provide enough information to the crew with adequate time to respond so that some situations can be avoided all together. A key component is the alerting philosophy used to direct the crew's attention to most critical information. No longer will the crew be solely responsible to deconflict alerts and determine the most critical hazard. In time-critical situations, the most appropriate solution is generated and presented, allowing the crew ample opportunity to execute an evasive maneuver. ANCOA provides a framework for integrating, organizing, prioritizing and displaying information for a new flight deck alerting system. The goal is to support prompt and appropriate responses to adverse conditions based on good situation awareness.

ANCOA was developed as a means to present alerting and notification information about conditions external to the aircraft. Specifically, the concept includes: (1) the ability to de-conflict currently separate alerts such as TCAS and GPWS; (2) the categorization (weather, traffic, ground, other) of alerts; (3) the prioritization (time-critical, tactical and strategic) of alerts to reduce pilot information processing 
requirements; (4) directional, multidimensional aural cueing to allow quick "pre-processing" of the condition (this aids in time-critical responses and in deciding the priority of the alerted condition relative to the on-going task); and (5) an integrated graphic presentation of conditions external to the aircraft to support better situation awareness.

ANCOA's integrated alerting framework was designed using a human-centered systems approach, which advocates that a system be designed to support those tasks required of the user. For example, in an emergency situation, the crew of an aircraft must detect that there is a problem, locate it, categorize the type of problem that it is, prioritize it by level of criticality, describe it at a deeper level to understand the nature of the problem, and finally respond quickly with an appropriate action. The alerting framework supports these six functions by specifying the display elements' design characteristics that promote the highest performance results. The framework specifies the alert prioritization and categorization schemes and presentation guidelines for displaying the information to the crew. Relevant information about critical and non-critical emerging conditions is presented to the crew to facilitate informed decisions based on good situation awareness of the problem space.

To examine the viability, usability and effectiveness of the framework and the ANCOA concept, a usability study was conducted at Honeywell Laboratories in Minneapolis, Minnesota. A summary of results from the usability evaluation is presented.

\section{USABILITY EVALUATION}

\section{Method}

We conducted a usability evaluation of the general integrated alerting framework, which included its application to the visual and auditory alerting displays, the alert categorization scheme, and the alert prioritization scheme. This concept was tested in Honeywell Laboratories' Flight Simulator. Nine commercial pilots with an average of 13,300 hours flight experience completed the $2 \frac{1}{2} 2$ hour evaluation. The goals of the study were to evaluate the display organization, configuration, ease-of-use, functionality, and navigation through the information within the prototype.

The pilots performed a card-sorting task to gain a better understanding of how pilots organize flight information. This task was intended to provide us with insight to help determine the appropriate categorization and prioritization for incoming information, such as traffic, icing, winds aloft, temperature, and gate status. The deck of cards contained 59 descriptors of information that could be encountered during a mission. The descriptors were based on a list derived by Riley et al. in an earlier NASA report (see Riley, et al., 1998) and are listed in the figure at the end of the paper. Pilots were given instructions to sort the cards into categories based on the similarity of the descriptors, and to provide names for the categories they created.

After completing of the card-sorting task, the pilots were taken to the flight simulator and given an introduction to the ANCOA concept, including an explanation of the Message Alerting and External Situation Awareness display (MAESA) and three-dimensional aural cueing. The MAESA display is the primary area for displaying time-critical, tactical, and strategic messages to the pilot visually. The display is a situation awareness, planning, and collaborative decisionmaking tool that allows pilots to view any flight phase and overlay information from multiple sources to gain an understanding of the current and future flight environments. The pilots were introduced to six different scenarios demonstrating the ANCOA functionality. For example, one scenario demonstrated the importance of priming pilots for potential hazards through the use of tactical alerts. While on the approach to the runway the pilot received a tactical report of windshear near the airport. Several minutes later, while landing the pilot received a windshear alert. Due to the advance information the pilot would be prepared to respond quickly.

Questionnaires followed each of the scenarios and after the entire block of trials. The experiment ended with a debriefing session to obtain detailed information about the pilots' preferences for the layout, functionality, and general usability of the concept.

\section{Results}

The data from the usability test came from output of the card-sorting task and the set of questionnaires that were given to the pilots. A "similarity matrix" was formed from the results of the card-sorting task. The similarity score for each pair of descriptors was the number of subjects that put the pair in the same category. The resulting similarity matrix was then subjected to multidimensional scaling (MDS) analysis and cluster analysis procedures.

MDS Analysis. The most notable statistic from a multidimensional scaling analysis is called a "stress level." The stress level of the test indicates the difficulty, or loss of information, in constraining the solution to a specific number of dimensions. A stress level of less than 0.15 is considered satisfactory for a MDS solution. In the current evaluation, the stress value for the two-dimensional MDS analysis was 0.08 , suggesting that a two-dimensional representation is adequate to explain the results. Note however, that with a card-sorting task such as this, there should, in theory, be approximately as many participants as cards. Given there were 59 cards and only 9 pilots in the current study, these results should be considered with caution. The MDS plot for the card-sorting task is shown in Figure 1.

Cluster Analysis. The results of the cluster analysis were consistent with those of the MDS analysis. In addition, the cluster analysis also identified subgroups not easily recognized in the MDS analysis. We associated the category labels that were generated by the pilots for their individually derived groups to the categories defined by the cluster and MDS analyses. The rules for grouping and naming the categories are detailed in Ververs, Good, Dorneich, Neihus, and Dewing (2000). The individual cards, category names and results of the cluster analysis and category title synthesis are presented below in Figure 2.

The card-sorting analysis identified three distinct categories of external conditions: (A) weather and weather-related hazards, (B) navigation, and (C) scheduling constraints. The weather and weather-related hazards category included ad- 
verse conditions encountered in-flight (e.g., wind shear) and on the ground (e.g., icy runway) as well as general weather information (e.g., dew point, visibility and wind speed). The navigation category covered general map elements (e.g., waypoint and radial) and hazards encountered while navigating (e.g., terrain, traffic). The third category was termed scheduling constraints. It includes the category many pilots labeled 'schedule,' 'crew information,' and 'no concern.' These three category labels will be applied to the ANCOA concept to differentiate, classify, and alert the crew of impeding hazards.



Figure 1. Two dimensional MDS solution for card-sorting task

Following the flight scenarios, the pilots were asked specific questions about the ANCOA framework and its implementation. Pilots indicated that they liked the strategic, tactical, and time-critical priority classification. They could easily direct their attention to the highest priority items based on the location of the alerts and color of the textual messages. ANCOA has a separate message area on the MAESA display where the textual alerts and notifications were integrated, prioritized and displayed. Pilots often found the area became too cluttered with multiple alerts, the messages were too long, and the text was too small. Furthermore, the pilots did not like the strategic information integrated with the time-critical alerts. In general, they found that the visual medium was appropriate for detailing the hazard information and for providing further information such as its specific location and its severity. Most pilots indicated that the aural cues were better at getting their attention, but the visual cues were better at addressing the threat. In addition, they found the use of the Primary Flight Display (PFD) to display time-critical alerts and the corresponding response to be very beneficial. Overall, the pilots indicated that ANCOA appeared to cover all the functions that they expected to see in such a concept and the information was grouped in a logical manner.
The results of the ANCOA usability evaluation indicated that pilots understood and approved of the strategic, tactical, and time-critical priority classification. Findings also suggested that a modification to the integration framework may be needed. Pilots endorsed the concept and believed the currently disparate alerts should be integrated into a single system. They believed the design improved their situation awareness. Pilots approved of the use of the Primary Flight Display for alerting of time-critical conditions and the associated response required. Other findings indicated that improvements were needed in the areas of directional audio cueing, weather and terrain graphics, navigation within the concept via the phase of flight arrangement, and the message area. See Ververs, et al. (2000) for more details. These improvements were made and ANCOA was subsequently modified. Figure 3 depicts the original MAESA, while Figure 4 is the updated version of the MAESA display, which addresses many of the issues raised by the pilots.

\section{CONCLUSIONS}

The findings from the usability evaluation of the ANCOA concept reveal several implications for advancements in the innovative IHAS products. In addition to the ability to integrate the information to prioritize and deconflict alerts, the overall alerting framework provides a means to graphically represent hazards by superimposing images to provide an integrated picture for better planning and overall situation awareness. This representation provides a means for inhibiting aural alerts but still provides a visual display of lower priority alerts.

A logic next step for integrated alerting systems is the creation of a system that integrates both internal and external conditions under a common framework. In many cases, onboard systems failures can change the performance characteristics of the aircraft resulting in unsafe interactions with external conditions. For instance, the loss of an engine induces pitch-limiting performance and therefore reduces the climb rate of an aircraft. This, in turn, can affect the crew's ability to clear mountainous terrain or avoid a converging aircraft. In other cases, the distinction between internal and external hazards is less obvious (e.g., icing). Only by integrating all the relevant information under a single framework will a crew's ability to respond to emergencies be fully supported. Achieving PANACEA will involve fully supporting Pilot Alerting and Notification of Adverse Conditions - Escape and $\underline{\text { Avoid- }}$ ance.

\section{ACKNOWLEDGMENTS}

NASA Langley Research Center supported this research. We wish to acknowledge and thank the technical monitors associated with the individual contracts: Paul Schutte and Anna Trujillo (NASA Contract NAS1-20219) and Drs. Chris and Celeste Belcastro (NASA Contract NAS1-00107). The authors would also like to thank Dr. Bill Rogers for his technical insights and contributions to this work, Pete Roeber and Jeff Rye for their skillful implementation of the concept, and Greg Niehus and Wende Dewing for interviewing the pilots. 


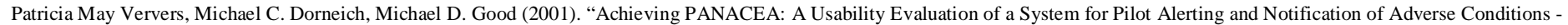
Escape and Avoidance", Proc. of the 45 ${ }^{\text {th }}$ Annual Meeting of the Human Factors and Ergonomics Society, Minneapolis/St. Paul, MN, October 8-12, 2001.

\section{REFERENCES}

Boucek, G. P., Hanson, D. C., Berson, B.L., Leffler, M. F., Po-Chedley, D. A., and Hendrickson, J. F. (1981). Aircraft alerting systems standardization study. AIAA/IEEE Digital Avionics Systems_Conference, AIAA: New York, 119- 128

Riley, V., DeMers, R., Good, M. Krishnan, K., Miller, C., and Misiak, C. (1998). Crew-centered flight deck alerting. NASA Technical Report Contract No. NAS1-20219. Hampton, VA: NASA Langley Research Center.
Ververs, P. M., Good, M. D., Dorneich, M. C., Niehus, G. and Dewing, W (2000). Alerting and Notification of Conditions Outside the Aircraft Usability Evaluation. NASA Technical Report Contract No. NAS100107. Hampton, VA: NASA Langley Research Center.

Ververs, P. M., Good, M. D., Rogers, W. H., Riley, V., and Dorneich, M. C. (September, 1999). Alerting and notification of conditions outside the aircraft: Concept defined and prototyped. NASA Contract No. NAS120219. Hampton, VA: NASA Langley Research Center.



Figure 2. The card clusters associated with the labels that pilots gave their groups 




Figure 3. Original Message Alerting and External Situation Awareness (MAESA) display

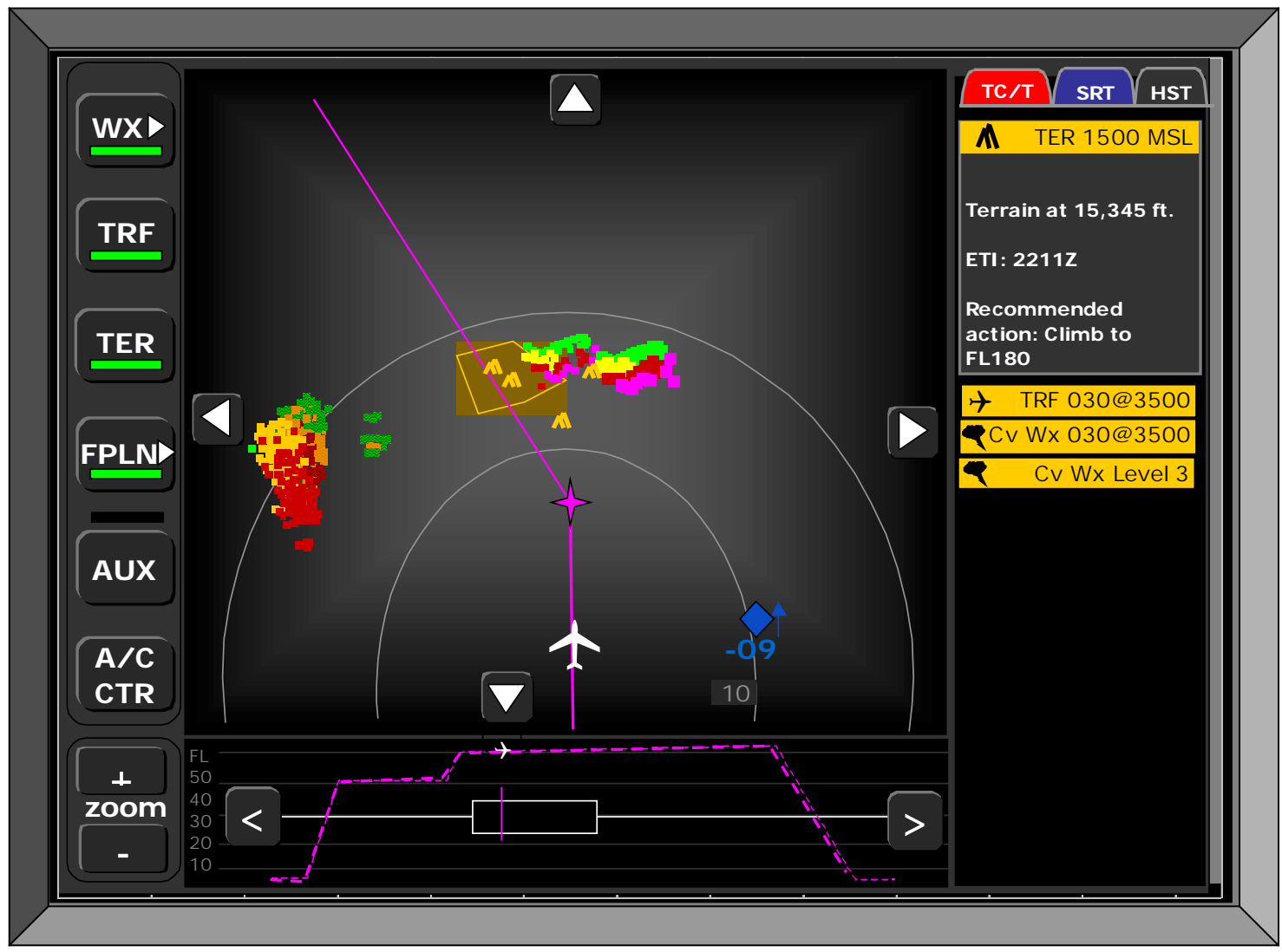

Figure 4. Refined Message Alerting and External Situation Awareness (MAESA) display 